\title{
Fracture toughness and crack-resistance curve behavior in metallic glass-matrix composites
}

\author{
M. E. Launey, ${ }^{1}$ D. C. Hofmann, ${ }^{2,3}$ J.-Y. Suh, ${ }^{2}$ H. Kozachkov, ${ }^{2}$ W. L. Johnson, ${ }^{2}$ \\ and R. O. Ritchie ${ }^{1,4^{*}}$ \\ ${ }^{1}$ Materials Sciences Division, Lawrence Berkeley National Laboratory, Berkeley, California, \\ 94720, USA
}

${ }^{2}$ Keck Laboratory of Engineering Materials, California Institute of Technology, Pasadena, California, 91125, USA

${ }^{3}$ Liquidmetal Technologies, Rancho Santa Margarita, California, 92688, USA

${ }^{4}$ Department of Materials Science and Engineering, University of California, Berkeley, California, 94720, USA

\begin{abstract}
:
Nonlinear-elastic fracture mechanics methods are used to assess the fracture toughness of bulk metallic glass (BMG) composites; results are compared with similar measurements for other monolithic and composite BMG alloys. Mechanistically, plastic shielding gives rise to characteristic resistance-curve behavior where the fracture resistance increases with crack extension. Specifically, confinement of damage by second-phase dendrites is shown to result in enhancement of the toughness by nearly an order of magnitude relative to unreinforced glass.
\end{abstract}

\footnotetext{
* Author to whom correspondence should be addressed. Electronic mail: roritchie@lbl.gov. URL: www.lbl.gov/Ritchie.
} 
The absence of microstructure in monolithic bulk metallic glasses (BMGs) can lead to marked strain localization and rapid shear-band propagation..$^{1,2}$ This effect, which causes extremely low macroscopic plastic deformability, can be devastating to mechanical performance in that properties that are limited by the extension of cracks, such as tensile ductility, toughness and fatigue resistance, can become severely compromised. Specifically, unstable fracture can ensue in monolithic BMGs along a single shear band with essentially zero macroscopic ductility ${ }^{3,4}$ such that the toughness is far lower than in comparable crystalline alloys. The essential element to developing high toughness in BMGs is to prevent single shear-band failures. By introducing a second phase in form of crystalline dendrites and by matching microstructural length scales (i.e., interdendritic spacing) to the mechanical length-scales (i.e., critical crack size for failure), nonlocalized plasticity in metallic glasses can be enhanced significantly. ${ }^{5-7}$ Indeed, the recent development of in situ bulk metallic glass-matrix composites has shown that provided this phase acts to arrest shear-band propagation over appropriate size-scales, ${ }^{8-10}$ the problems of poor ductility, toughness and fatigue resistance can be mitigated.

One problem here is that the new composite BMGs, are undoubtedly far tougher than monolithic BMGs or some earlier BMG composites, and current processing methods often cannot make section sizes large enough to meet the 
critical validity requirements for accurate fracture mechanics measurements, i.e., not meeting fracture-mechanics requirements for valid stress intensity $\mathrm{K}$ - or $J$ dominated crack-tip fields and/or for plane-strain constraint. Indeed, many toughness measurements on BMG materials reported in the literature ${ }^{11-15}$ are inaccurate due to problems of inappropriate measurement techniques (e.g., the area under a compression stress/strain curve), absence of sharp stress concentrators (e.g., using a relatively blunt notch rather than a fatigue pre-crack) and insufficient test-sample size. Moreover, while single-value measurements, such as $K_{\mathrm{Ic}}$, properly define the toughness of nominally brittle materials, they can be insufficient for alloys displaying extensive plastic deformation and subcritical cracking, as can occur in many BMG composites. Stable crack growth in metallic glasses is not generally observed and has not been reported for monolithic metallic glasses. However, the new metallic glass-matrix composite materials exhibit considerable plasticity and thus a resistance-curve (R-curve) evaluation is warranted.

In this study, we use a nonlinear-elastic fracture mechanics (J-integral $\left.{ }^{16}\right)$ characterization, including R-curve determination, ${ }^{17}$ of the toughness of in situ metallic glass-matrix composites to accurately measure the fracture resistance of these alloys, to separate the critical crack-driving forces for crack initiation and 
growth, and to interpret behavior in terms of the prevalent mechanisms of microstructural damage and toughening.

BMG composites (DH1 and DH3 alloys) containing a ductile dendritic phase were prepared in situ by relatively rapid cooling of homogeneous

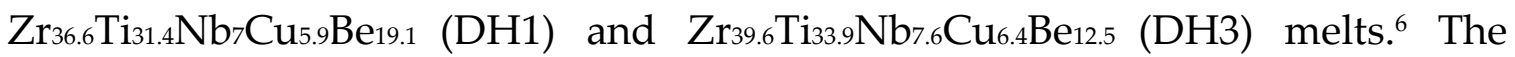
alloys were semi-solidly processed ${ }^{8}$ to give uniform two-phase microstructures with coarse second-phase dendrites (Fig. 1). The DH1 and DH3 microstructures comprise, respectively, 42 and $67 \mathrm{vol} \% \%$ of the latter (bcc) $\beta$-phase (interdendritic spacings $\sim 5$ and $2 \mu \mathrm{m}$ with compositions of $\mathrm{Ti}_{40} \mathrm{Zr}_{45} \mathrm{Nb}_{14} \mathrm{Cu}_{1}$ and $\mathrm{Ti}_{45} \mathrm{Zr}_{40} \mathrm{Nb}_{14} \mathrm{Cu}_{1}$ ) within a $\mathrm{Zr}_{34} \mathrm{Ti}_{22} \mathrm{Nb}_{2} \mathrm{Cu}_{9} \mathrm{Be}_{33}$ and $\mathrm{Zr}_{34} \mathrm{Ti}_{17} \mathrm{Nb}_{2} \mathrm{Cu}_{9} \mathrm{Be}_{38}$ matrix. ${ }^{8}$ The resulting alloys display high tensile ductilities (>10\%) with yield strengths of exceeding $1 \mathrm{GPa} .{ }^{8}$

Single edge-notched bend SE(B) specimens were machined out of ingots with thicknesses $B$ of $4.2-6.0 \mathrm{~mm}$ and widths $W$ of $9.2-10.5 \mathrm{~mm}$. For comparison, similar R-curve tests were performed on the monolithic metallic glass Vitreloy 1 $\left(\mathrm{Zr}_{41.2} \mathrm{Ti}_{13.8} \mathrm{Cu}_{12.5} \mathrm{Ni}_{10} \mathrm{Be}_{22.5}\right)$, and $17-7 \mathrm{PH}$ precipitation-hardened stainless steel (AISI-631), heat treated to match the ductility, yield and tensile strength of the composites.

R-curves were measured in terms of the J-integral to evaluate the fracture resistance as a function of crack extension, $\Delta a$, under a monotonically increasing 
loading. SE(B) specimens were fatigue pre-cracked, and then loaded under displacement control $(15 \mu \mathrm{m} / \mathrm{s})$ in three-point bending in a servo-hydraulic testing machine in accordance with ASTM standards. ${ }^{18}$ After the onset of cracking, crack extension was monitored from the elastic unloading load-line compliance ${ }^{19}$ and verified with electrical-potential drop measurements. Provided all validity criteria ${ }^{18}$ were met, $K$-based fracture toughness values were backcalculated from the $J$ measurements using the standard mode I $J-K$ equivalence, $K_{\mathrm{J}}=\left(J \cdot E /\left(1-v^{2}\right)\right)^{0.5}$.

Resulting R-curves in terms of the equivalent stress intensity $K_{\mathrm{J}}$ demonstrate the extensive crack-growth resistance of the composite BMG materials, compared to monolithic Vitreloy 1 which exhibits no rising R-curve (Fig. 2). Whereas immediate unstable fracture occurs at a stress intensity of $54 \mathrm{MPa} \sqrt{\mathrm{m}}$ in Vitreloy 1, in the respective DH1 and DH3 alloys R-curves are initially steep (from cracktip blunting), followed by stable cracking over $\sim 1.5 \mathrm{~mm}$ until a steady-state "plateau" is reached at 155 and $200 \mathrm{MPa} \sqrt{\mathrm{m}}$. This means that by these measures, the composites are $\sim 3-4$ times tougher than their unreinforced matrices. Results for stainless steel are shown for comparison. However, these data are limited by the specimen-size requirements for $J$-dominance. According to ASTM Standards, ${ }^{18}$ the maximum $J$ capacity for a specimen is given by the smaller of $J_{\max }$ $=b \sigma_{\mathrm{y}} / 10$ or $B \sigma_{\mathrm{y}} / 10$ ( $\sigma_{\mathrm{y}}$ is the flow stress, and $b(=W-a)$ is the uncracked ligament) 
and crack extension cannot exceed $\Delta a_{\max }=0.25 b$; R-curve data outside these limits are invalid (Fig. 2). As the size of both DH1 and DH3 samples met this criteria, i.e., $b, B \geq 2.6 \mathrm{~mm}$, we can accurately ascribe the fracture toughness of the DH1 alloy to be $J_{\mathrm{Q}}=J_{\mathrm{Ic}}=96 \mathrm{~kJ} / \mathrm{m}^{2}$, equivalent to a $K_{\mathrm{Jc}}$ of $97 \mathrm{MPa} \sqrt{\mathrm{m}}$, and the DH3 alloy to be $J_{\mathrm{Q}}=J_{\mathrm{Ic}}=282 \mathrm{~kJ} / \mathrm{m}^{2}$, equivalent to a $K_{\mathrm{Jc}}$ of $157 \mathrm{MPa} ل_{\mathrm{m}}$; all values can be considered to be valid as per ASTM standards. The corresponding size of the 17$7 \mathrm{PH}$ stainless steel samples also met all ASTM criteria, i.e., $b, B \geq 1.5 \mathrm{~mm}$, such that $J_{\mathrm{Q}}=J_{\mathrm{Ic}}=74 \mathrm{~kJ} / \mathrm{m}^{2}$.

It is important to note here that unless section sizes of $50 \mathrm{~mm}$ or more can be made, which is not a feasible proposition at present, LEFM parameters such as

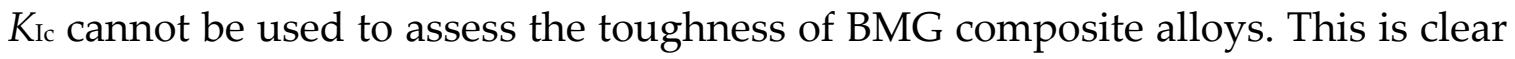
from Fig. 3a which shows the extent of gross plasticity after $J-R$ testing. Specifically, to measure the $157 \mathrm{MPa} \sqrt{\mathrm{m}}$ toughness in the $\mathrm{DH} 3$ alloy would require a 52-mm thick specimen with a width twice this dimension. ${ }^{18}$ With a $J$ based analysis, section sizes essentially met validity; the current $K_{\mathrm{Jc}}$ values are thus deemed to be accurate and representative of the toughness of these materials. Consequently, in comparison to monolithic BMG alloys, where $K_{\mathrm{Ic}}$ can be as low as $20 \mathrm{MPa} \sqrt{\mathrm{m}},{ }^{20-22}$ we can now state with assurance that these metallic glass-matrix composites exhibit toughnesses that are significantly higher than for 
monolithic BMGs, and further are comparable to the toughest crystalline metallic alloys at these high strength levels (>1 GPa).

During steady-state crack growth, the increase in fracture resistance is associated with the formation of an extensive plastic zone (Fig. 3a) and a wide distribution of damage around the crack tip (Fig. 3b). Plastic deformation occurs via the development of highly organized patterns of regularly spaced shear bands distributed uniformly along the crack path; these bands initiate and propagate in the glassy matrix until they are blocked by second-phase dendrites (Fig. 3c). Due to the strong matrix/dendrite interface, slip is transferred from the matrix to the dendrites wherein secondary shear bands are initiated in other directions to accommodate the strain (Fig. 3d).

The key to developing the high toughness is to prevent single shear-band failures. This is achieved through confinement of the shear bands as a result of the mismatch in plastic response. In earlier versions of such composite alloys, cooling rate variations within the ingots caused large differences in the overall dendrite length-scale with interdendrite spacings varying by two orders of magnitude (from $\sim 1$ to $100 \mu \mathrm{m}$ ). ${ }^{6,23}$ As noted above, the characteristic microstructural length-scale, the spacing between dendrite arms, has to match the critical mechanical length-scale, the crack size for fracture, so that single shear bands are arrested before they can become long enough to cause failure. 
The consequence of this is the formation of multiple shear bands which substantially enhances plasticity with a resulting beneficial effect on toughness. When the interdendritic spacing is too large, this interaction does not occur and the resulting toughening is minimal. This can be appreciated from previous

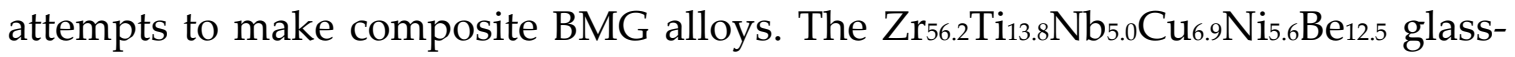
matrix composite LM2 alloy, for example, contained too small a volume fraction of dendrites with a spacing as large as $\sim 10 \mu \mathrm{m} ;{ }^{;, 24}$ this alloy displayed resistancecurve behavior and was tougher than the unreinforced metallic glass matrix ( $K_{\text {Ic }}$ $\sim 15 \mathrm{MPa} \sqrt{\mathrm{m}})$, but its fracture toughness was still only $31 \mathrm{MPa} \sqrt{\mathrm{m}} . .^{24} \mathrm{By}$ constraining the initial deformation band to arrest over a smaller length-scale $(\sim 2$ $\mu \mathrm{m})$, as with the present DH3 alloy, a efficient multiple shear bad formation leads to extensive plastic shielding and fracture toughness up to $200 \mathrm{MPa} \sqrt{\mathrm{m}}$, almost one order of magnitude higher than the monolithic metallic glass.

Supported by the Office of Science, Office of Basic Energy Sciences, Division of Materials Sciences and Engineering, of the U.S. Department of Energy under Contract No. DE-AC02-05CH11231 (for MEL, ROR), with funding for DCH and WLJ from the Office of Naval Research. DCH acknowledges financial support from the Department of Defense via a NDSEG fellowship. 

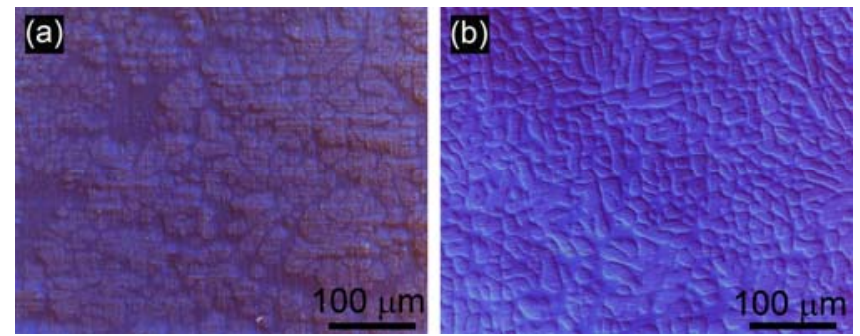

FIG. 1. Z-contrast optical micrographs of the microstructure of (a)

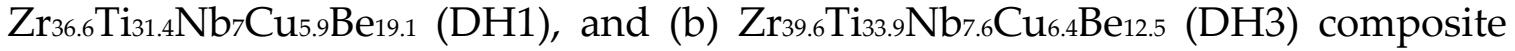
BMG alloys. 


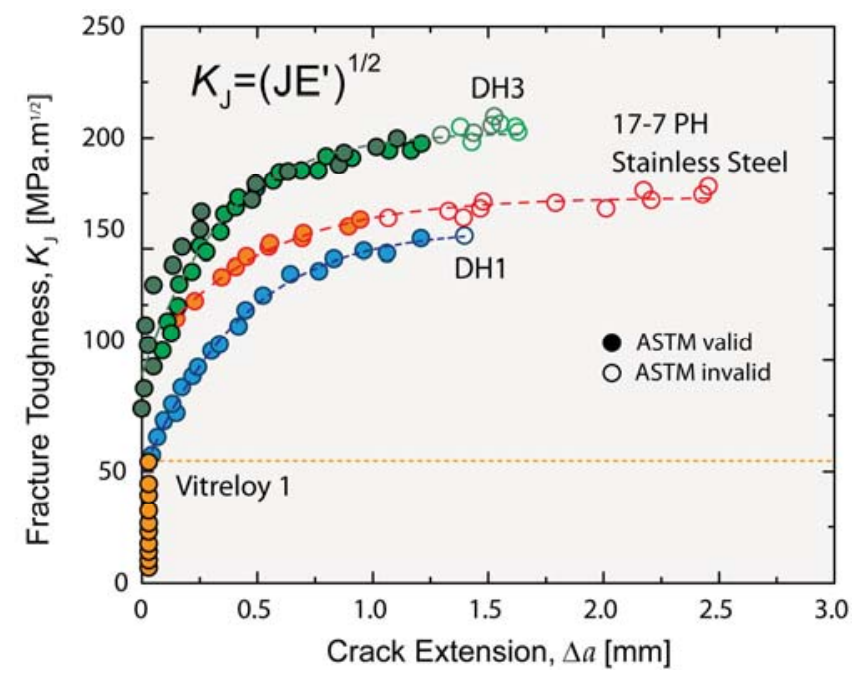

FIG. 2. R-curves showing resistance to fracture in terms of the stress intensity, $K_{\mathrm{J}}$, as a function of crack extension, $\Delta a$, for the DH1 and DH3 composite BMG, monolithic Vitreloy 1 BMG and a 17-7 PH stainless steel. 

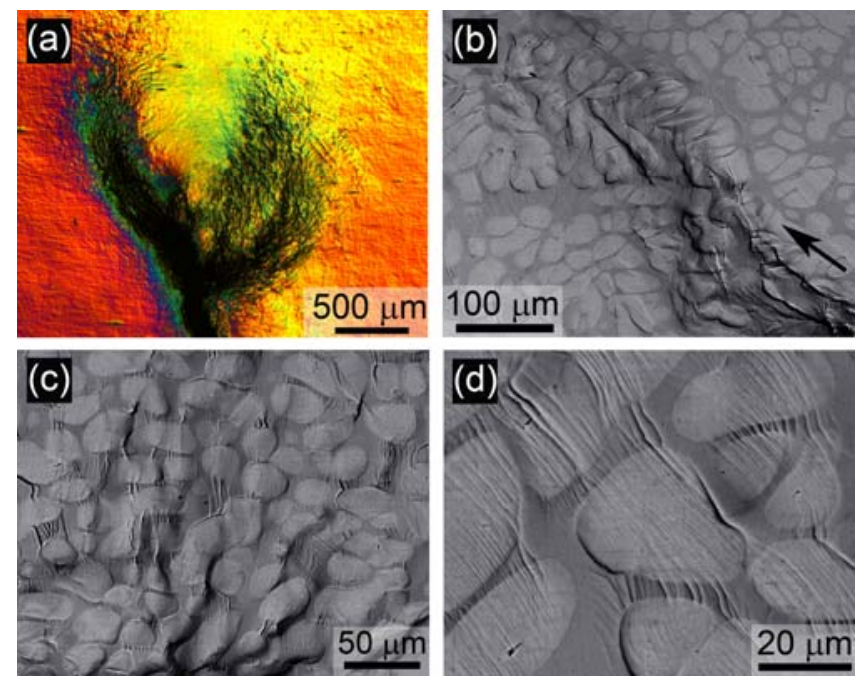

FIG. 3. (a) Differential interference contrast (Nomarski) micrograph of DH3 composite alloy showing extensive plasticity around the crack tip of the order of several millimeters. (b) SEM backscattered electron image showing wide distribution of damage ahead of the crack tip in the DH1 alloy. The arrow indicates the direction of crack propagation. (c) Deformation in DH3 occurs through the development of highly organized patterns of regularly spaced shear bands distributed uniformly along the crack path. (d) Microcracks in DH3 are nucleated along the shear bands or at the matrix/dendrite interface. 